\title{
Method for 3D Object Reconstruction Using Several Portions of 2D Images from the Different Aspects Acquired with Image Scopes Included in the Fiber Retractor
}

\author{
Kohei Arai \\ Graduate School of Science and Engineering \\ Saga University \\ Saga City, Japan
}

\begin{abstract}
Method for 3D object reconstruction using several portions of 2D images from the different aspects which are acquired with image scopes included in the fiber retractor is proposed. Experimental results show a great possibility for reconstruction of acceptable quality of 3D object on the computer with several images which are viewed from the different aspects of $2 \mathrm{D}$ images.
\end{abstract}

Keywords-3D image reconstruction; fiber retractor; image scope.

\section{INTRODUCTION}

Medical surgery is possible through a not so large hole using medical surgery instruments such as fiber retractor, image scope, etc. It is called Laparoscopic surgery [1]-[3]. Damage due to Laparoscopic surgery is much smaller than the typical medical surgery with widely opened human body and retracts the nidus in concern. In order to make a medical surgery plan, 2D images which are derived from "image fiber scope" are used usually. It is not easy to make a plan because 2D images are not enough. Medical doctor would like to see 3D image of objects entirely. On the other hand, fiber retractor contains not only one fiber scope but also several fibers can be squeezed in one tube (acceptable size of the human body hole). The image fiber scope which is proposed here is containing several fibers in one tube. Anoptical entrance is attached at each tip of the fiber. The several fibers are aligned along with fiber retractor. Therefore, 2D images are acquired with the different fiber image scopes. It is also possible to reconstruct $3 \mathrm{D}$ object image using the acquired 2D images with the several fiber image scopes.

Simulation studies are conducted with simulation data of 2D images which are derived from fiber image scopes. 3D object image is reconstructed successfully with an acceptable image quality. The following section describes the proposed Laparoscopic surgery with the fiber image scopes which are aligned along with fiber retractor followed by simulation studies. In the process, geometric calibration is highly required for the system together with a high fidelity of 3D image reconstruction. Finally, conclusion and some discussions are described.

\section{Proposed Laparoscopic SURgery With The Fiber IMAGE SCOPES Which ARE ALIGNED ALONG With FIBER RETRACTOR}

\section{A. Laparoscopic surgery}

Illustrative view of the laparoscopic surgery is shown in Fig.1.Laparoscopy output of 2D images is monitored by computer display in a real time basis. Looking at the monitor display image medical surgery is operated with surgical instruments. Thus a portion of the nidus of survival lottery is removed with retractor.

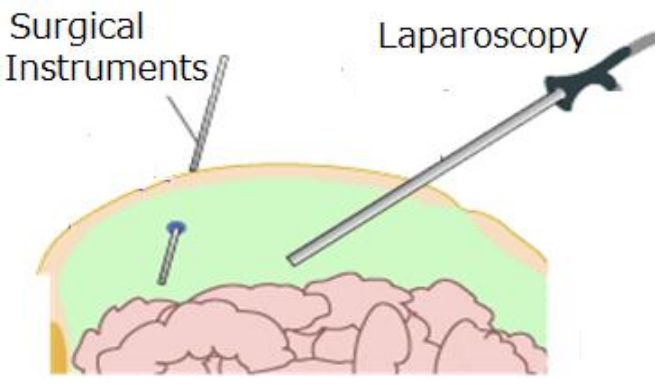

Figure 1. Illustrative view of Laparoscopic surgery

In order to make a surgery plan, 3D images of the nidus containing survival lottery is highly required.3D images can be reconstructed with several 2D images acquired from the different aspects. 2D images are acquired with image scope.

\section{B. Image Scope}

Outlook of the image scope is shown in Fig.2. Fig.2 (a) shows the fiber optical entrance of the image scope while Fig.2 (b) shows aft-optics of the image scope. Although Fig.2 shows just one of fiber image scope, the proposed system includes several fiber image scopes into one fiber tube.

Thus 2D images from the different aspects can be acquired with the several fiber image scopes. Then $3 \mathrm{D}$ image is reconstructed on the computer using the acquired $2 \mathrm{D}$ images. 


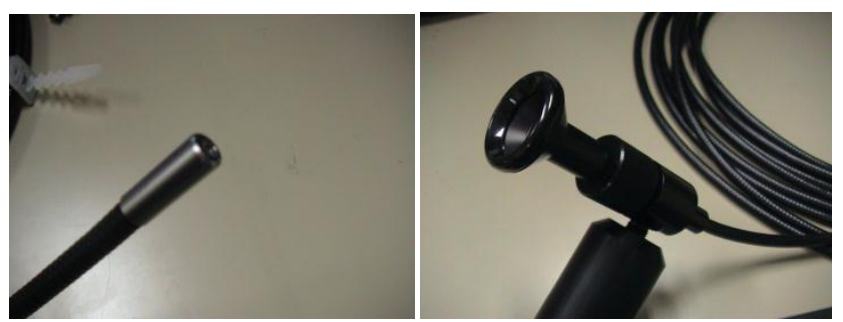

(a) Tip of fiber image scope (b)Outoptics of fiber image scope

Figure 2. Outlook of the image scope

\section{Fiber Retractor}

The aforementioned several fiber image scopes into one fiber tube are shown in Fig.3. Namely, optical entrances of 8 fiber image scopes into one fiber tube, in this case, are aligned along with circle shape of fiber ring. Original shape of this fiber ring is just a line. As shown in Fig.4, fibers in the fiber tube are closed loop shape at the begging. This is called fiber retractor hereafter. After the line shaped fiber retractor is inserted into human body, the tips of fibers are expanded. The shape of fiber tips becomes circle from the line. Thus the tips of the fiber of which optical entrance and light source aft-optics are attached are aligned as shown in Fig.3. This is called Fiber Retractor with Image Scopes: FRIS.

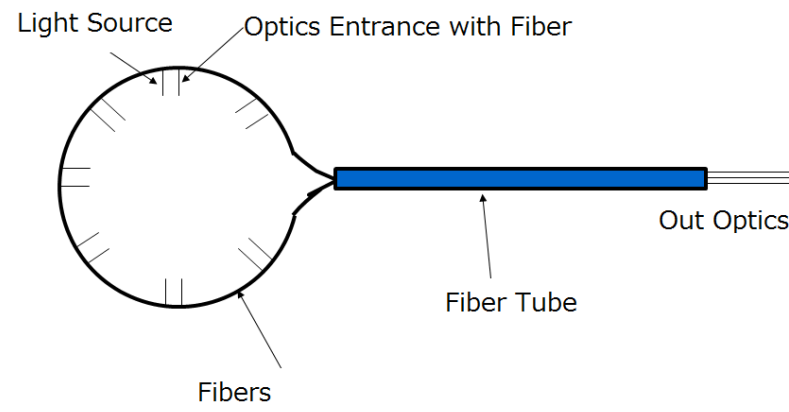

Figure 3. Proposed fiber retractor with image scopes for 3D image acquisitions

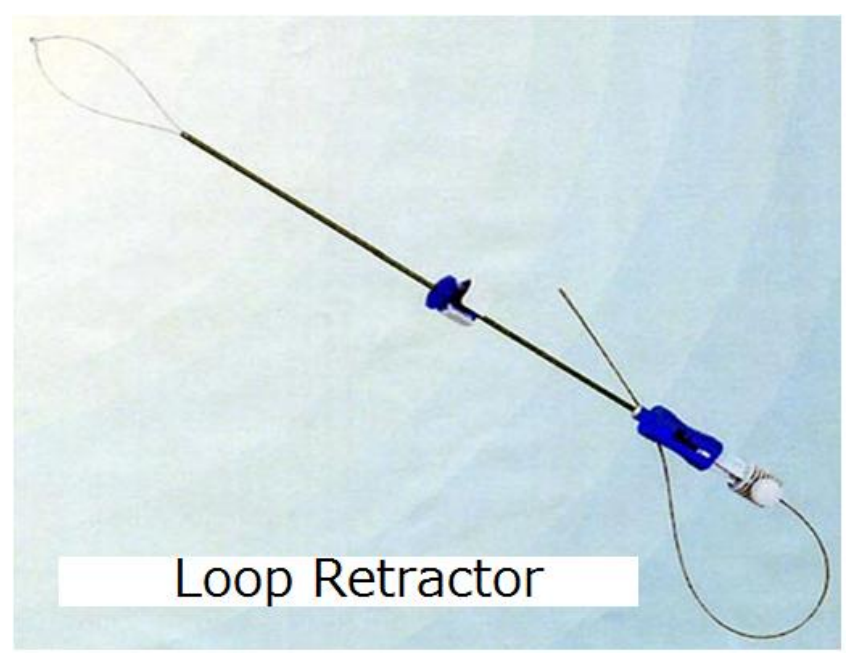

Figure 4. Example of fiber retractor

Using FRIS, 3D object image is acquired as shown in Fig.5. Fig.5 (a) shows how to acquire 3D object (Red sphere) while Fig.5 (b) shows examples of acquired 2D images with $60 \%$ of overlapping between two adjacent 2D images acquisition locations.

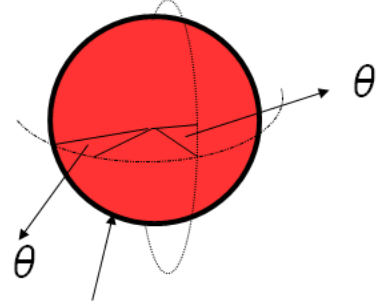

Object

(a)3D object image acquisition method $60 \%$ Overlap

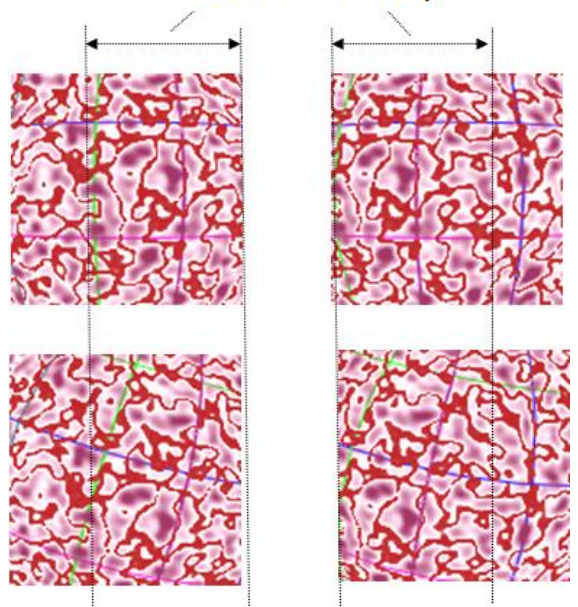

(b)Method for 2D images acquisition with $60 \%$ of overlapping ratio between two adjacent $2 \mathrm{D}$ image acquisition locations

Figure 5. Method for 3D object image acquisitions

\section{Camera Calibrations}

Object coordinate [X Y Z 1]t can be converted to 2D image coordinate $[\mathrm{XdYd} 1] \mathrm{t}$ as shown in equation (1).

$$
H c\left[\begin{array}{c}
X d \\
Y d \\
1
\end{array}\right]=\left[\begin{array}{llll}
C_{11} & C_{12} & C_{13} & C_{14} \\
C_{21} & C_{22} & C_{23} & C_{24} \\
C_{31} & C_{32} & C_{33} & C_{34}
\end{array}\right]\left[\begin{array}{c}
X \\
Y \\
Z \\
1
\end{array}\right]
$$

where $\left[C_{i j}\right]$ is called camera parameter. The camera parameter can be determined by camera calibration. It, however, is difficult to calibrate camera geometry in human body. Therefore, camera calibration is used to be conducted in laboratory in advance to the $3 \mathrm{D}$ object image acquisition. In the camera calibration, 2D images, $\mathrm{A}$ and $\mathrm{B}$ which are acquired from the two different locations are used. Thus four equations can be obtained as shown in equation (2).

$$
\begin{aligned}
& C_{A 11} X+C_{A 12} Y+C_{A 13} Z+C_{A 14}=C_{A 31} X X d_{A}+C_{A 32} Y X d_{A}+C_{A 33} Z X d_{A}+C_{A 34} X d_{A} \\
& C_{A 21} X+C_{A 22} Y+C_{A 23} Z+C_{A 24}=C_{A 31} X Y d_{A}+C_{A 32} Y Y d_{A}+C_{A 33} Z Y d_{A}+C_{A 34} Y d_{A} \\
& C_{B 11} X+C_{B 12} Y+C_{B 13} Z+C_{B 14}=C_{B 31} X X d_{B}+C_{B 32} Y X d_{B}+C_{B 33} Z X d_{B}+C_{B 34} X d_{B} \\
& C_{B 21} X+C_{B 22} Y+C_{B 23} Z+C_{B 24}=C_{B 31} X Y d_{B}+C_{B 32} Y Y d_{B}+C_{B 33} Z Y d_{B}+C_{B 34} Y d_{B}
\end{aligned}
$$

Using these equations, all the camera parameters are determined based on least square method. 


\section{E. Process Flow of the Proposed 3D Image Reconstructions}

Fig.6 shows the process flow of the proposed 3D image reconstruction with FRIS. First, 2D images are acquired from the different aspects surrounding of the 3D object in concern. Then geometric feature is extracted from the 2D images for tie point matching. Because the two adjacent $2 \mathrm{D}$ images are acquired with $60 \%$ of overlapping ratio, 3D image can be reconstructed using these $2 \mathrm{D}$ images with reference to $3 \mathrm{D}$ space coordinate. Thus $3 \mathrm{D}$ shape is reconstructed. Then $2 \mathrm{D}$ images are mapped onto the 3D image surfaces and rendering is applied to the reconstructed 3D shape.

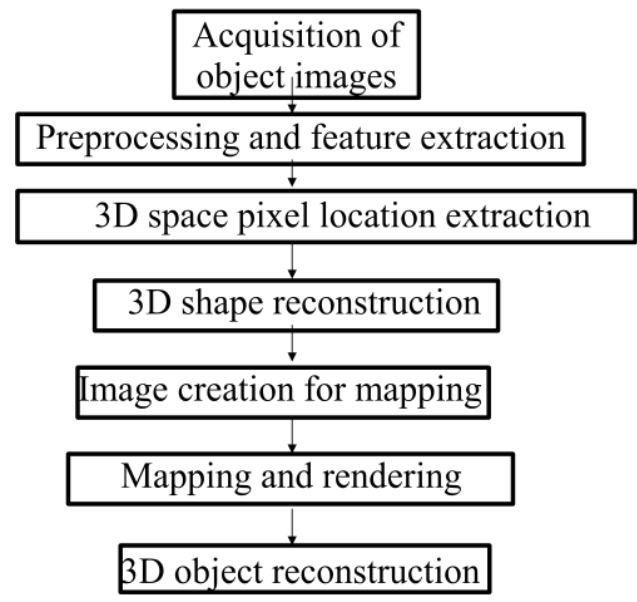

Figure 6. Process flow of the proposed 3D reconstruction with 2D images acquired with FRIS.

2D images for mapping are created as shown in Fig.7. Namely, corresponding 3D image coordinate is calculated with the pixels on the 2D image coordinate. From now on, spherical shape of object is assumed to be $3 \mathrm{D}$ object shape.

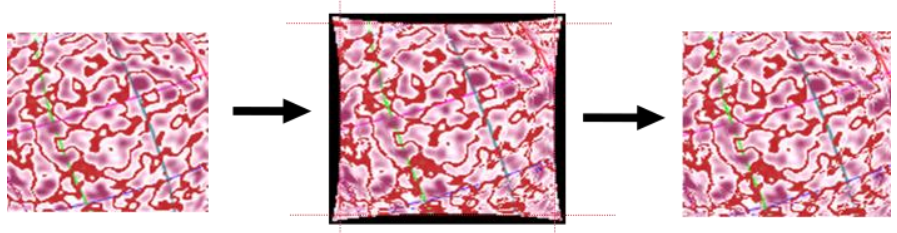

(a)acquired 2D image (b)geometric converted image (c)2D image for mapping

Figure 7. Creation of 2D images for mapping.

In this process, [ $\mathrm{x} 1 \mathrm{y} 1 \mathrm{1}$ ] $\mathrm{t}$ coordinate pixel location is converted to $[\mathrm{x} 2 \mathrm{y} 2$ 1]tpixel location through Affine transformation. Translation and rotation parameters are determined with the corresponding pixel locations between two adjacent 2D images as shown in Fig.8.

Examples of rotation converted images with the different rotation angles are shown in Fig.9. $\left[\begin{array}{l}x_{2} \\ y_{2}\end{array}\right]=k\left[\begin{array}{ccc}\cos \phi & -\sin \phi & x t \\ \sin \phi & \cos \phi & y t\end{array}\right]\left[\begin{array}{c}x_{1} \\ y_{1} \\ 1\end{array}\right]$
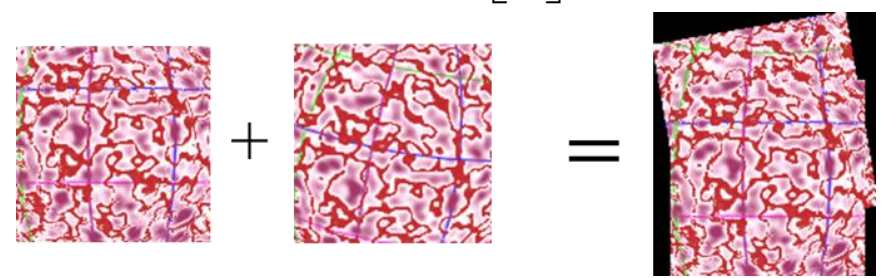

Figure 8. Rotation and translation is applied to the acquired $2 \mathrm{D}$ adjacent images.

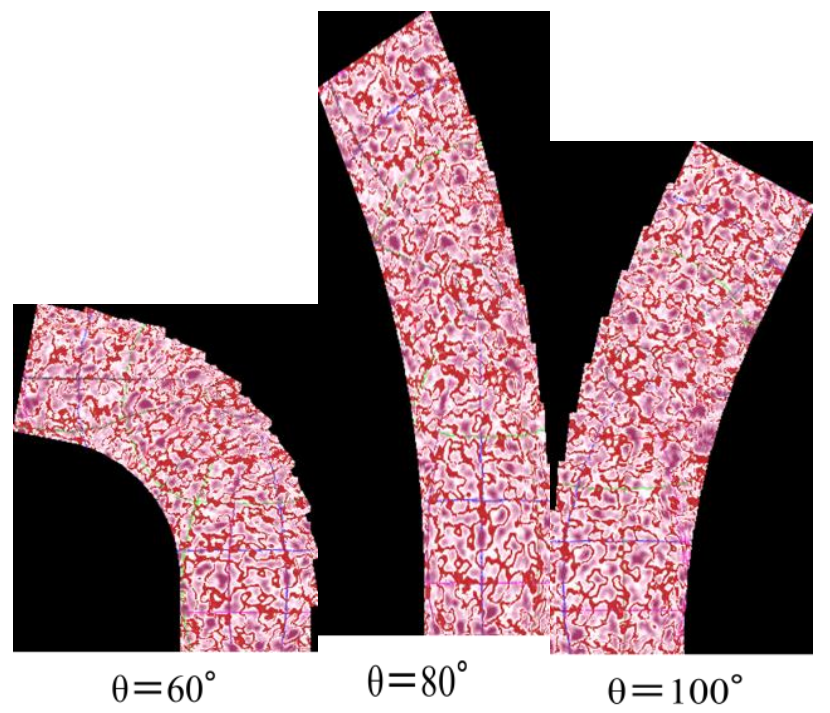

Figure 9. Rotation conversions with the different angles.

In this process, the number of tie points is important because mapping accuracy depends on the number of tie points. Lattice points on the $2 \mathrm{D}$ image coordinates are selected as tie points as shown in Fig.10.

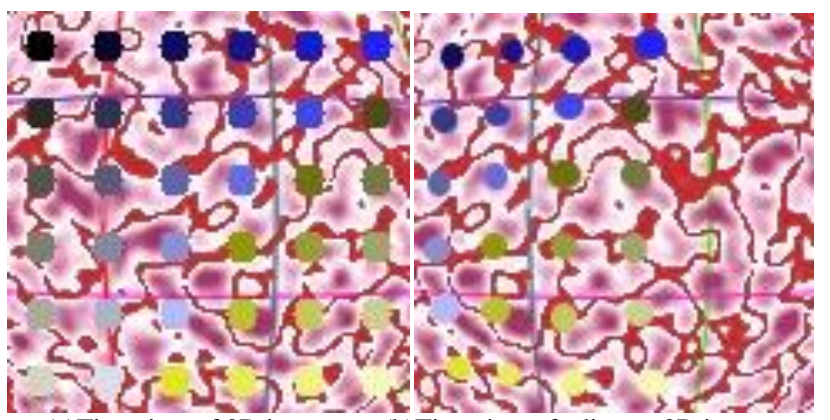

(a)Tie points of 2D image (b)Tie points of adjacent 2D image

Figure 10. Tie points (corresponding points between two adjacent 2D images)

Figure 11 shows how to combine two adjacent two image strips into one 2D image for mapping. In this process, the corresponding pixel locations are referred in between in the two adjacent $2 \mathrm{D}$ images. 

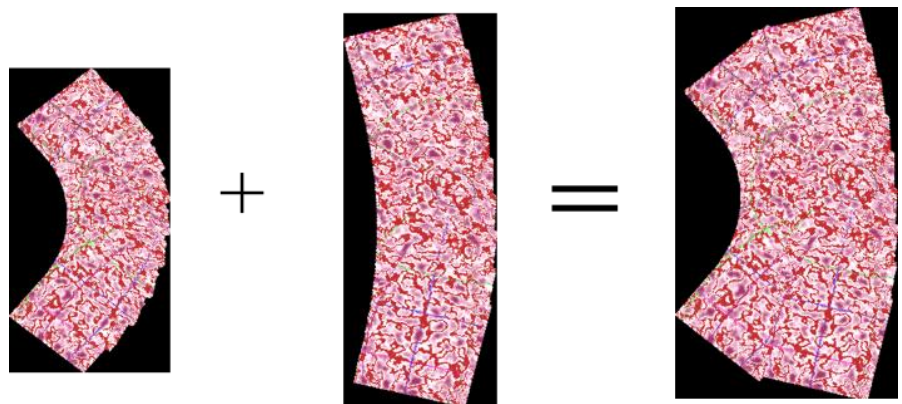

Figure 11. Method for combine two adjacent 2D images

\section{F. Texture Mapping}

UV mapping method is applied to the 2D mapping images as a texture mapping. Namely, image coordinate system is converted to the mapped 3D image coordinate system, UV coordinate. 3D object shape is converted to the top and bottom view of the UV coordinate systems as shown in Fig.12. Fig.13 shows the examples of the top and bottom view of the mapping images

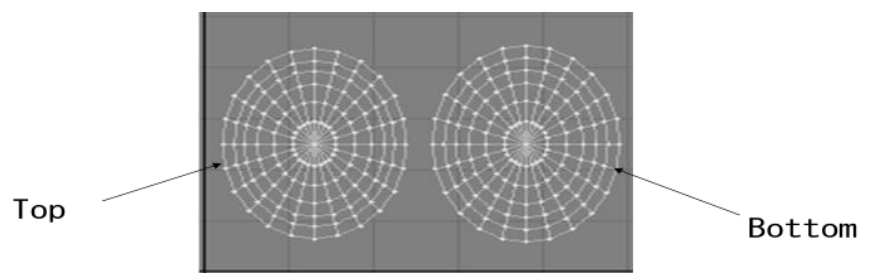

Figure 12. UV coordinate for the top and bottom view

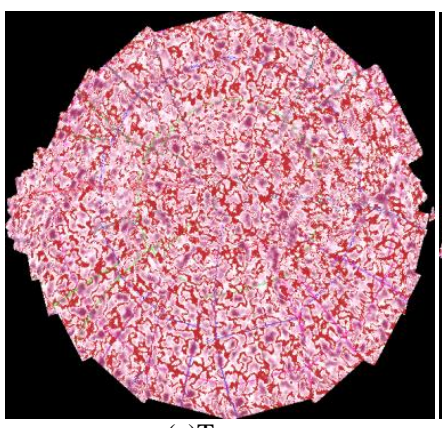

(a)Top

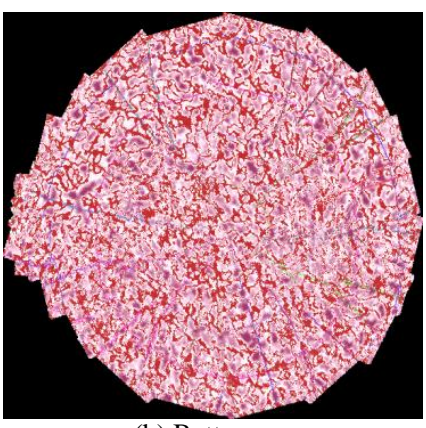

(b) Bottom
Figure 13. Examples of the top and bottom view of the mapping images.

\section{G. Rendering}

Finally, rendering is conducted and displayed onto computer screen as shown in Fig.14. Thus 3D object image is reconstructed in the computer. As shown in Fig.15, rendering has to be made with smooth surface as much as it could be. Fig.15 (a) shows a potion of 3D object surfaces while Fig.15 (b) shows side view of the reconstructed 3D object image. Although the textures of the two adjacent 2D images have to be matched each other, both texture patterns do not match perfectly due to mapping error derived from coordinate conversion. Therefore, some smoothing process has to be applied as post processing.

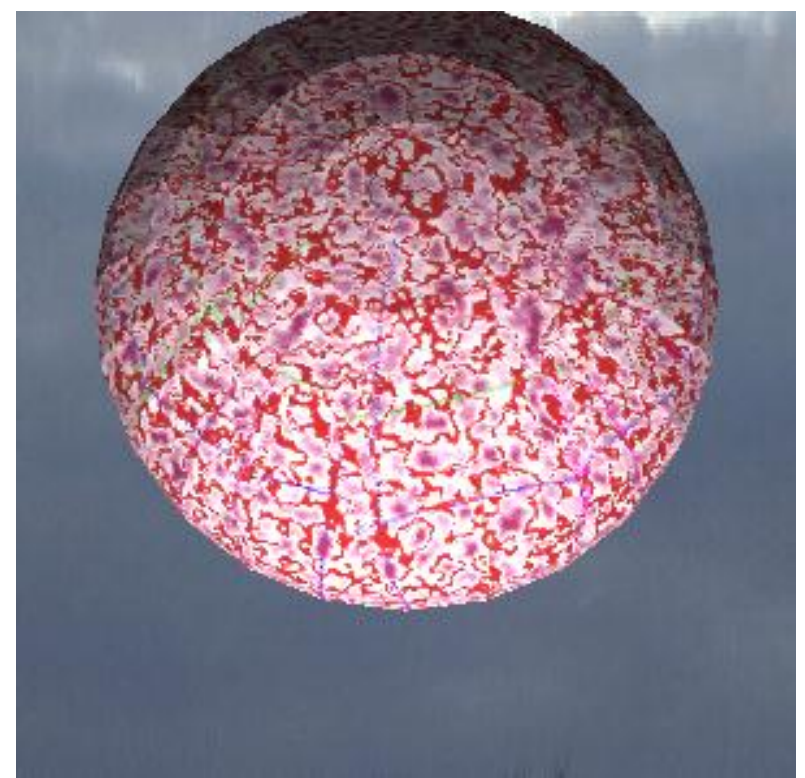

Figure 14. Reconstructed 3D object image displayed onto computer screen.

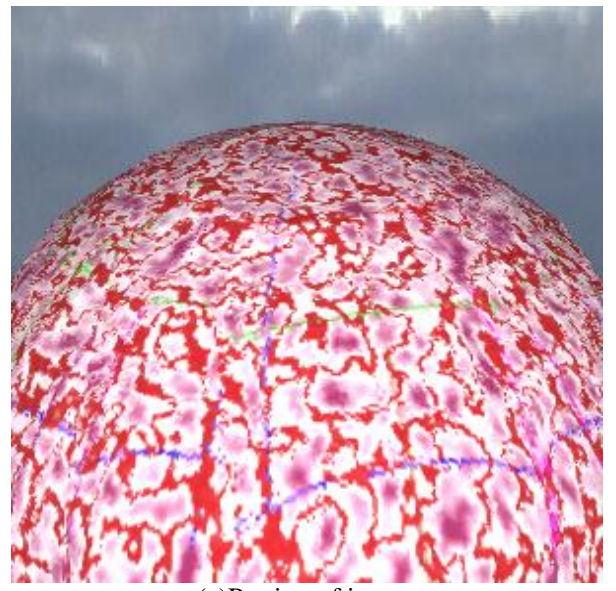

(a)Portion of image

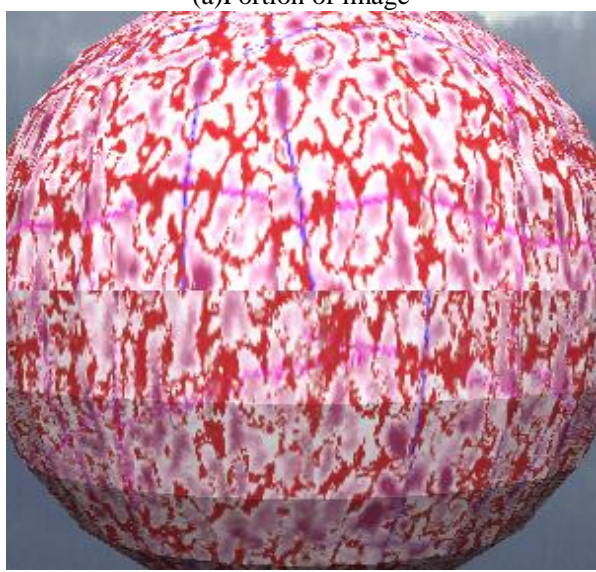

(b)Side view

Figure 15. Example of the reconstructed 3D object image 


\section{EXPERIMENTS}

Using LightWave3D software tool, a simulation study is conducted. $10 \mathrm{~cm}$ of diameter of sphere with surface texture is assumed to be an object. Light source is situated at the same location with camera. Camera of which focal length is $33.8 \mathrm{~mm}$ with aperture angle of 25 degree is used for simulation study. The distance between the camera and the 3D object is $20 \mathrm{~cm}$. When the $3 \mathrm{D}$ object is acquired with the camera, the cameras are assumed to be aligned along with the circle with every 20 degree of angle. Therefore, $60 \%$ of overlapping 2D image acquisition can be done. Corresponding points for tie point matching are extracted manually.

Fig.16 shows the simulation result with the aforementioned procedure. At the top left of Fig.16 shows top view while the bottom left shows front view of the reconstructed 3D object images. Meanwhile, the top right of Fig.16 shows oblique view while the bottom right of Fig.16 shows side view of the reconstructed 3D object. All these images are reasonable.
This representation of 3D object image is specific to the LightWave3D software tool. Another example is shown in Fig.17. If the lattice point locations are given for the top view, front view, and side view, then 3D object image is appeared on the top right of the window of the computer screen. Even if the real 3D object image is complex shape and texture as shown in Fig.18, the proposed method may create $3 \mathrm{D}$ object image onto computer screen.

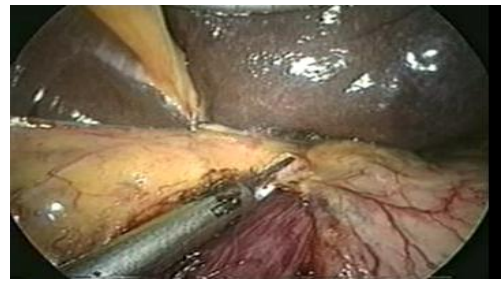

Figure 16. Figure 18 Real 3D object image

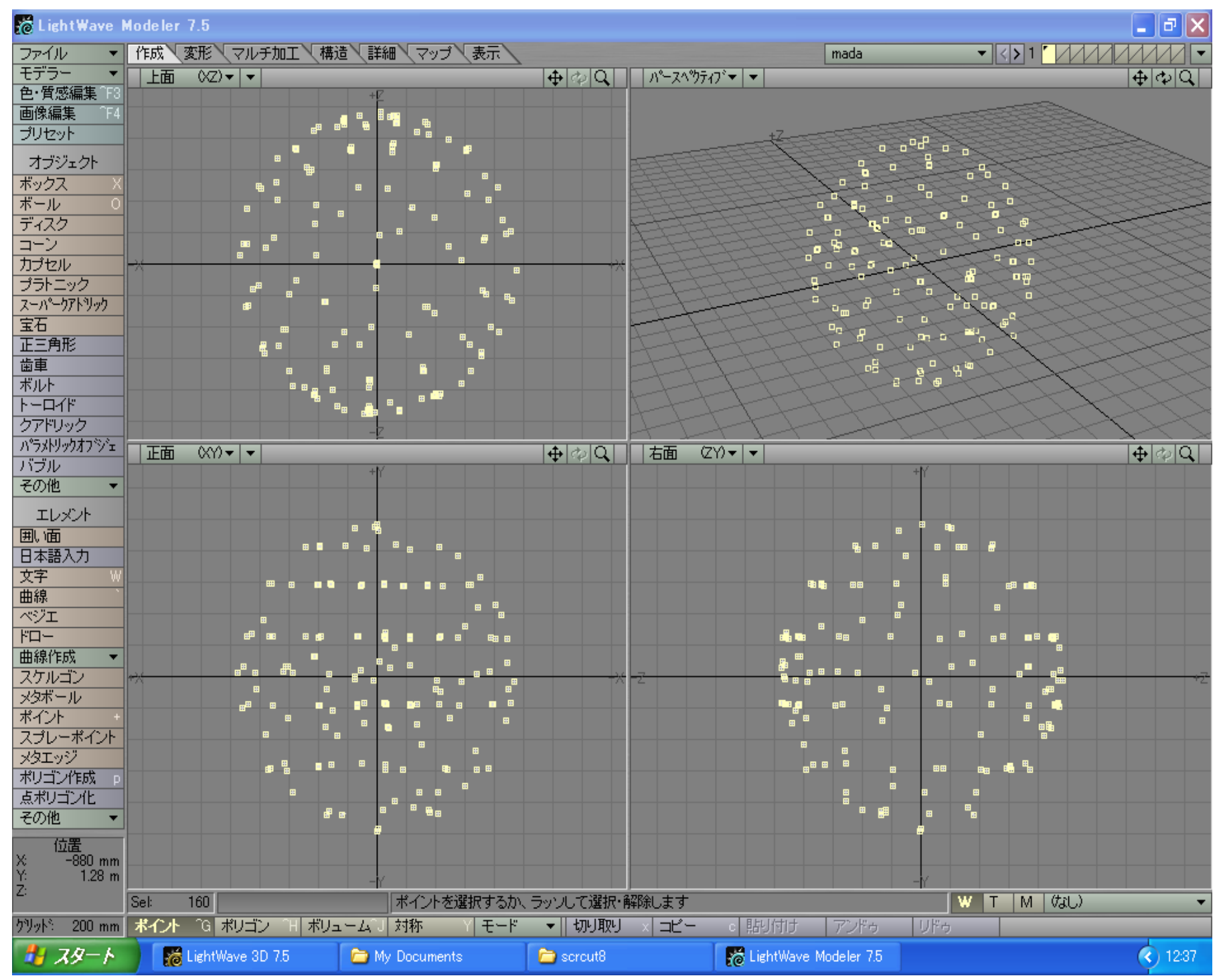

Figure 17. Reconstructed 3D object image as a simulation study. 


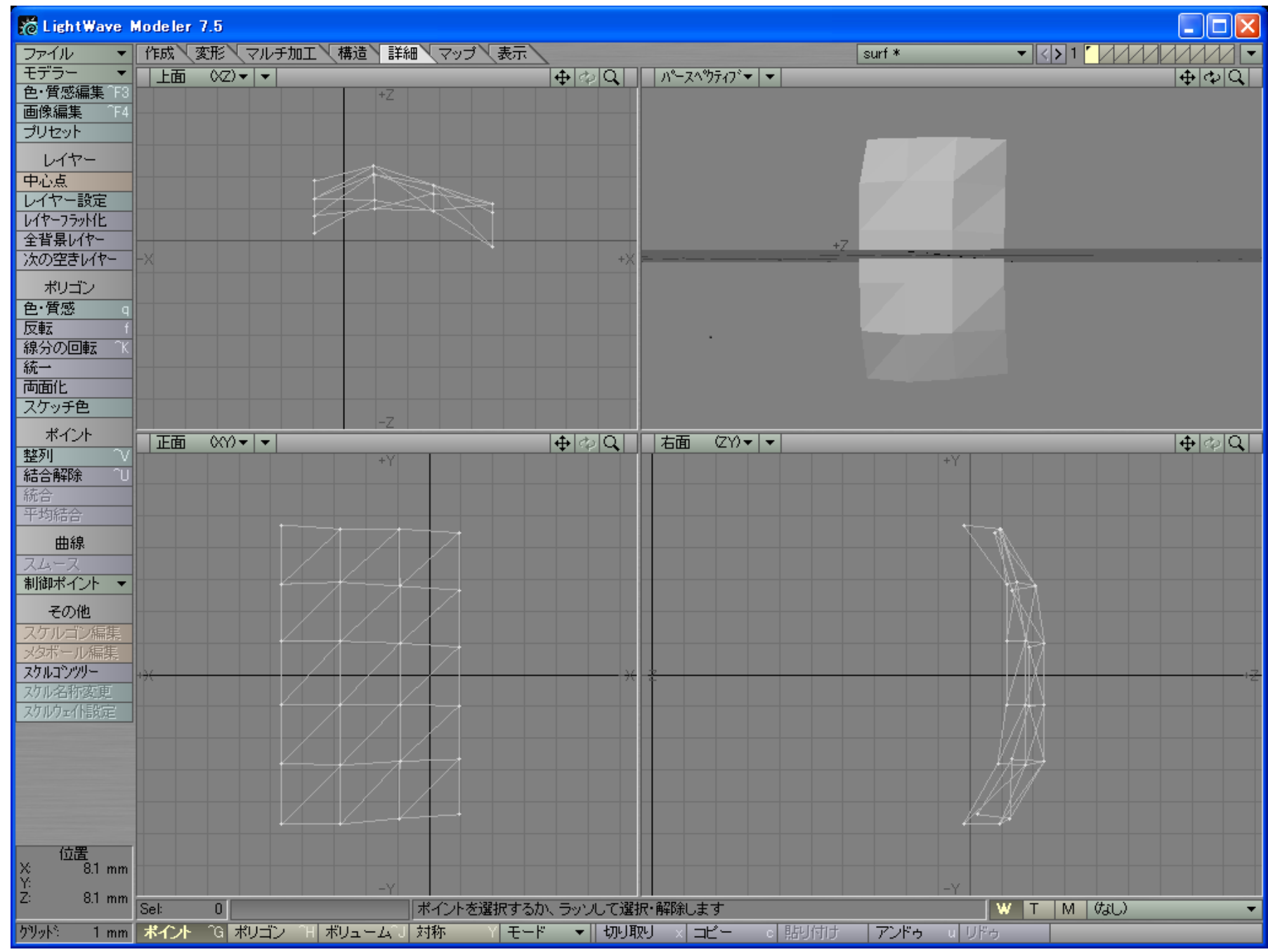

Figure 18. Figure 17Sub-window assignments for the top view, the front view, the side view and the reconstructed 3D object image for LightWave3D software tool

\section{CONCLUSION}

Method for 3D object reconstruction using several portions of $2 \mathrm{D}$ images from the different aspects which are acquired with image scopes included in the fiber retractor is proposed. Experimental results show a great possibility for reconstruction of acceptable quality of 3D object on the computer with several images which are viewed from the different aspects of $2 \mathrm{D}$ images.

Further investigations are highly required for making smooth texture surfaces between two adjacent 2D images.

\section{ACKNOWLEDGMENT}

The author would like to thank Mr. Junji Kairada for his effort to creation of simulation images.

\section{REFERENCES}

[1] Mastery of Endoscopic and Laparoscopic Surgery W. Stephen, M.D. Eubanks; Steve Eubanks (Editor); Lee L., M.D. Swanstrom (Editor);
Nathaniel J. Soper (Editor) Lippincott Williams \& Wilkins 2nd Edition 2004

[2] Clarke HC (April 1972). "Laparoscopy-new instruments for suturing and ligation". Fertil. Steril. 23 (4): 274-7

[3] Walid MS, Heaton RL (2010). "Laparoscopy-to-laparotomy quotient in obstetrics and gynecology residency programs". Arch Gyn Ob 283 (5): $1027-1031$.

\section{AUTHORS PROFILE}

Kohei Arai, He received BS, MS and PhD degrees in 1972, 1974 and 1982, respectively. He was with The Institute for Industrial Science, and Technology of the University of Tokyo from 1974 to 1978 also was with National Space Development Agency of Japan (current JAXA) from 1979 to 1990. During from 1985 to 1987, he was with Canada Centre for Remote Sensing as a Post Doctoral Fellow of National Science and Engineering Research Council of Canada. He was appointed professor at Department of Information Science, Saga University in 1990. He was appointed councilor for the Aeronautics and Space related to the Technology Committee of the Ministry of Science and Technology during from 1998 to 2000. He was also appointed councilor of Saga University from 2002 and 2003 followed by an executive councilor of the Remote Sensing Society of Japan for 2003 to 2005 . He is an adjunct professor of University of Arizona, USA since 1998. He also was appointed vice chairman of the Commission "A" of ICSU/COSPAR in 2008. He wrote 30 books and published 332 journal papers 